\title{
Evaluation of a multiplex PCR for bacterial pathogens applied to bronchoalveolar
} lavage

\author{
K. Strålin*, J. Korsgaard ${ }^{\#}$ and P. Olcén"
}

ABSTRACT: The present study assessed the diagnostic usefulness of a multiplex PCR (mPCR) for Streptococcus pneumoniae, Haemophilus influenzae, Mycoplasma pneumoniae and Chlamydophila pneumoniae applied to bronchoalveolar lavage (BAL).

Fibreoptic bronchoscopy was performed on 156 hospitalised adult patients with lower respiratory tract infection (LRTI) and 36 controls. BAL fluid was analysed with bacterial culture and MPCR.

By conventional diagnostic methods, S. pneumoniae, $\boldsymbol{H}$. influenzae, $M$. pneumoniae and $\mathbf{C}$. pneumoniae were aetiological agents in 14, 21, 3.2 and $0 \%$ of the LRTI patients, respectively. These pathogens were identified by BAL mPCR in 28, 47, 3.2 and $0.6 \%$ of cases, respectively, yielding sensitivities of $86 \%$ for S. pneumoniae, $88 \%$ for $\boldsymbol{H}$. influenzae, $100 \%$ for $\mathbf{M}$. pneumoniae and $0 \%$ for $C$. pneumoniae, and specificities of $81,64,100$ and $99 \%$ for S. pneumoniae, $H$. influenzae, M. pneumoniae and C. pneumoniae, respectively. Of the 103 patients who had taken antibiotics prior to bronchoscopy, S. pneumoniae was identified by culture in $2.9 \%$ and by $\mathrm{mPCR}$ in $31 \%$. Among the controls, $\mathrm{mPCR}$ identified S. pneumoniae in $11 \%$ and $H$. influenzae in $39 \%$.

In lower respiratory tract infection patients, bronchoalveolar lavage multiplex PCR can be useful for identification of Streptococcus pneumoniae, Mycoplasma pneumoniae and Chlamydophila pneumoniae. The method appears to be particularly useful in patients treated with antibiotics.

KEYWORDS: Bronchoalveolar lavage, Haemophilus influenzae, lower respiratory tract infection, Mycoplasma pneumoniae, PCR, Streptococcus pneumoniae

$\mathrm{n}$ cases of severe community-acquired lower respiratory tract infection (LRTI), it is desir-

able to identify the pathogen causing the infection in order to choose appropriate antibiotic treatment. Cultures from the lower respiratory tract are often used because blood cultures have low sensitivity for LRTI aetiology [1]. As sputum cultures may be contaminated by the oropharyngeal flora, so fibreoptic bronchoscopic techniques have been developed to enable collection of lower respiratory tract samples with minimal risk of contamination. At present, a major reason for fibreoptic bronchoscopy (FOB) in LRTI patients is failure to respond as expected to the first-line antibiotic treatment $[2,3]$. However, PRATS et al. [4] demonstrated that the culture yield of Streptococcus pneumoniae and Haemophilus influen$z a e$ is rapidly reduced in lower respiratory tract secretions during antibiotic treatment; because of this, PRATs et al. [4] and other groups [5, 6] have suggested that FOB should be performed earlier in the course of LRTI, if possible prior to antibiotic treatment.
In contrast to culture, PCR is not dependent on viable bacteria. In a study by WHEELER et al. [7], PCR for S. pneumoniae often remained positive in sputum during antibiotic treatment, while sputum culture quickly became negative. Consequently, PCR applied to bronchoscopic samples may be useful for identification of $S$. pneumoniae and $H$. influenzae during antibiotic treatment. To the present author's knowledge, this has not been evaluated.

One reason for nonresponse to $\beta$-lactam antibiotics in LRTI is atypical pathogens as a cause of the infection. While PCR for Legionella spp. applied to bronchoalveolar lavage (BAL) fluid has been found useful [8], PCR for Mycoplasma pneumoniae and Chlamydophila pneumoniae has not been evaluated in BAL samples. However, as sputum samples have been shown to be more useful than upper respiratory tract samples for PCR identification of M. pneumoniae $[9,10]$ and $C$. pneumoniae [11], and because sputum samples can often not be collected in patients with LRTI,

\section{AFFILIATIONS}

Depts of *Infectious Diseases, and "Clinical Microbiology, Örebro University Hospital, Örebro, Sweden. \#Dept of Chest Diseases, Aarhus University Hospital, Aalborg, Denmark.

CORRESPONDENCE

K. Strålin

Dept of Infectious Diseases

Örebro University Hospital

SE-70185 Örebro

Sweden

Fax: 4619184855

E-mail: kristoffer.stralin@orebroll.se

Received:

January 162006

Accepted after revision:

May 092006

\section{SUPPORT STATEMENT}

This study was supported by grants from the Research Committee of Örebro County Council (Sweden). 
BAL PCR could probably be useful for identification of these two pathogens in LRTI patients.

The current authors have developed a multiplex PCR (mPCR) for simultaneous identification of $S$. pneumoniae, $H$. influenzae, M. pneumoniae and C. pneumoniae in respiratory tract samples. The method showed an analytical sensitivity of $100 \%$ (89 out of 89 ) and a specificity of $99 \%$ (167 out of 168) when 257 bacterial strains (37 different species) were tested [12]. mPCR has been evaluated on sputum samples and nasopharyngeal samples from adults with community-acquired pneumonia (CAP) and controls with promising results [13].

The aim of the present study was to estimate the diagnostic accuracy of mPCR applied to BAL samples from adult patients with LRTI. For further determination of the specificity of BAL mPCR, a control group including patients investigated on suspicion of malignancy was included.

\section{MATERIALS AND METHODS}

\section{Study subjects}

The present study was performed prospectively at the Silkeborg County Hospital, Silkeborg, Denmark, in its Dept of Internal Medicine, which has 100 beds. Silkeborg County Hospital is the only hospital in the region and serves a basic population of 100,000 inhabitants in the area. As previously described [14], all immunocompetent adult patients hospitalised for LRTI during weekdays between September 1997 and August 2000 were consecutively included in the present study. Thus, the included patients were unselected adult patients with LRTI requiring hospitalisation. The clinical diagnosis of LRTI required that the patient had fever (rectal temperature $\geqslant 37.6^{\circ} \mathrm{C}$ within $48 \mathrm{~h}$ of inclusion in the study) and/or an increased leukocyte count $\left(\geqslant 11 \times 10^{9}\right.$ leukocytes $\left.\cdot \mathrm{L}^{-1}\right)$ in peripheral blood on admission, together with increased focal symptoms from the lower airways with at least one of three newly developed symptoms of increased dyspnoea, increased coughing and/or increased sputum purulence. Patients with known malignancy and patients with oxygen saturation $<85 \%$ with a maximum of $1 \mathrm{~L}$ nasal oxygen were not included. Chest radiograph and spirometry were performed. Demographic, comorbidity and smoking data were collected, and information on prior antibiotic treatment for any diagnosis was obtained from either the individual patient or the patient's general practitioner.

Adult patients who consecutively underwent FOB for suspected malignancy were enrolled as controls. Information about prior antibiotic treatment and smoking data were collected.

\section{Bronchoscopy}

All patients and controls underwent a standardised FOB with BAL [15] within $24 \mathrm{~h}$ of admission. Prior to the FOB, the mouth and pharynx were anaesthetised with a solution of $4 \%$ lidocaine. The fibre bronchoscope was introduced through the nose or through the mouth and additional anaesthetics $(1 \%$ lidocaine solution) were applied as the scope passed the larynx and trachea. A sterile, thin tube was introduced in the working channel of the bronchoscope. The tip of the scope was wedged in an appropriate segment in the bronchus, and thereafter lavage was performed. A segment of bronchus affected by a new pulmonary infiltrate, as seen at a prior chest radiograph, was chosen for lavage. In the LRTI patients without radiographically observed infiltrates and the control patients, the middle lobe was chosen for lavage. Between one and three portions of $60 \mathrm{~mL}$ of isotonic sodium chloride solution were used for lavage and the aspirated fluid was collected in one single portion for microbiological analyses.

\section{Conventional microbiological investigations}

BAL fluid and sputum samples from the LRTI patients and BAL fluid from the controls were analysed with culture at the Dept of Clinical Microbiology, Aarhus University Hospital (Aalborg, Denmark), within a maximum of $6 \mathrm{~h}$ from the time of sampling. The specimens were cultured on $5 \%$ horse blood agar and chocolate agar with semiquantitative determinations by dispersion of 1 and $10 \mu \mathrm{L}$ on each half of the plate. The plates were incubated in $5 \%$ carbon dioxide at $35^{\circ} \mathrm{C}$ for $24-$ $48 \mathrm{~h}$. Bacterial identification was performed according to standard microbiological methods [16]. The cut-off limit for a positive BAL or sputum culture result was $10^{2}$ colony-forming units $(\mathrm{cfu}) \cdot \mathrm{mL}^{-1}$ sample. After culture, the BAL fluid was frozen at $-20^{\circ} \mathrm{C}$.

BAL samples and throat swabs, which were placed in two sucrose phosphate chlamydial transport media, from the LRTI patients where sent to the Dept of Bacteriology, Mycology and Parasitology, Statens Serum Institute (Copenhagen, Denmark), and were analysed with singleplex PCR (sPCR) assays for $M$. pneumoniae, C. pneumoniae, Chlamydophila psittaci, and Legionella spp. $[17,18]$. In addition, the BAL samples and throat swabs were cultured for C. pneumoniae [19].

From the LRTI patients, blood samples were collected for culture with a Bactec blood-culturing system (BioMérieux, Marcy-lEtoile, France) at the Dept of Clinical Microbiology, Aarhus University Hospital. Nonfrozen urine samples were sent to the Dept of Bacteriology, Mycology and Parasitology, Statens Serum Institute, and were analysed for pneumococcal capsular polysaccharides by countercurrent immunoelectrophoresis [20], and for Legionella pneumophila serogroup 1 antigen by enzyme immunoassay (Biotest AG, Dreieich, Germany).

\section{Multiplex PCR}

Frozen BAL samples from the LRTI patients and controls were sent to the Dept of Clinical Microbiology, Örebro University Hospital (Örebro, Sweden), for mPCR. A detailed description of the development and procedures of the MPCR is presented in a previous paper [12]. mPCR was performed under blinded conditions for simultaneous identification of specific genes for S. pneumoniae (lytA, 229 bp), M. pneumoniae (P1, 483 bp), C. pneumoniae (ompA, $368 \mathrm{bp})$, and $H$. influenzae (16S rRNA, $538 \mathrm{bp}$ ). The following primers were used: lyt $A$, 5'-CGGACTACCGCCTTTATATCG-3' and 5'-GTTTCAATCGTCAAGCCGTT-3'; P1, 5'-ACTCGGAGGACAATGGTCAG-3' and 5'-CAAACCCGGTCTTTTCGTTA-3'; ompA, 5'-ACACGATGCAGAGTGGTTCA-3' and 5'-TGTTTACAGAGAATTGCGATACG-3'; and 16SrRNA, 5' -TCCTAAGAAGAGCTCAGAGAT-3' and $5^{\prime}$-TGATCCAACCGCAGGTTCC-3'. The concentration of all primers was $50 \mu \mathrm{M}$. Briefly, DNA from 0.2-0.5 mL BAL fluid was extracted using the automatic MagNa Pure LC DNAIsolation system (Roche Diagnostics, Mannheim, Germany). 
Extracted DNA $(10 \mu \mathrm{L})$ was added to a $40-\mu \mathrm{L}$ mixture to give a total $\mathrm{mPCR}$ mixture volume of $50 \mu \mathrm{L}$, which included $90 \mathrm{nM}$ of each of the two lytA primers and $250 \mathrm{nM}$ of each of the other six primers, $800 \mu \mathrm{M}$ of the deoxyribonucleoside triphosphate mixture (Perkin-Elmer Applied Biosystem, Norwalk, CT, USA), $1.5 \mathrm{U}$ of AmpliTaq Gold DNA Polymerase (Perkin-Elmer Applied Biosystem), and $10 \times$ PCR buffer (Perkin-Elmer Applied Biosystem). PCR amplification was performed on a GenAmp PCR system 9600 (PerkinElmer Applied Biosystem) with the following parameters: $94^{\circ} \mathrm{C}$ for $10 \mathrm{~min}$ followed by 40 cycles at $94^{\circ} \mathrm{C}$ for $30 \mathrm{~s}, 58^{\circ} \mathrm{C}$ for $30 \mathrm{~s}$, and $72^{\circ} \mathrm{C}$ for $1 \mathrm{~min}$, with the last cycle concluding with $72^{\circ} \mathrm{C}$ for $7 \mathrm{~min}$, before storage at $4^{\circ} \mathrm{C}$. The amplified sample was examined by electrophoresis on an agarose gel containing ethidium bromide. The result was compared with a DNA molecular weight marker. In every PCR run, one negative control and positive controls for each one of the four investigated bacteria were included. Samples positive for the 16S rRNA gene were considered positive for $H$. influenzae if they were also positive in a verification PCR for the P6 gene, using the same PCR protocol. The P6 PCR mixture included $125 \mathrm{nM}$ of each $P 6$ primer (5'-TTGGCGGWTACTCTGTTGCT-3' and 5'-TGCAGGTTTTTCTTCAC(GT-3'). All mPCR-negative samples were spiked with a reference strain of $S$. pneumoniae and were re-run by the PCR protocol for control of inhibition.

By serial dilution of bacterial strains, the detection levels of the mPCR have been shown to be $10^{2} \mathrm{cfu} \cdot \mathrm{mL}^{-1}$ of sample for $S$. pneumoniae and $H$. influenzae, and $10^{3}$ genome copies or inclusion-forming units per millilitre of sample for $M$. pneumoniae and C. pneumoniae [12].

\section{Criteria for aetiologies and exclusion of aetiologies}

The criteria for LRTI aetiologies used in the present study were as follows. S. pneumoniae: positive blood culture, positive urinary antigen test, positive BAL culture, or positive sputum culture; H. influenzae: positive blood culture, positive BAL culture, or positive sputum culture; M. pneumoniae: positive BAL or throat swab SPCR; C. pneumoniae: positive BAL or throat swab sPCR, or positive BAL or throat swab culture. These criteria were used in the calculations of sensitivities and specificities of BAL mPCR. If no available reference analysis was positive for a pathogen, the pathogen was ruled out as an aetiological agent in the specificity analysis.

\section{Ethics}

The study was performed according to the Declaration of Helsinki II and was approved by the local ethical committee; all participating patients gave written consent.

\section{RESULTS \\ Patients and controls}

During the study period, 167 patients fulfilled the study criteria for LRTI. Eight patients declined participation, thus 159 patients were included in the prospective study. BAL fluid from 156 patients was available for $\mathrm{MPCR}$ and these patients were included in the present study. The LRTI patients had a median age of 63 yrs (range 26-90 yrs), 48 (31\%) patients were current and $62(40 \%)$ were previous smokers, and the mean pack-yrs history in the current and previous smokers was 27 yrs. A chronic lung disease was documented in $72(46 \%)$ patients, and the mean forced expiratory volume in one second on day 7 from inclusion was $58 \%$ of predicted. New infiltrates were identified by radiography in 87 (56\%) LRTI patients. The median interval between illness onset and FOB was 8 days; FOB was performed within 2 weeks of illness onset in $72 \%$ of the patients, and FOB was performed within $24 \mathrm{~h}$ of hospitalisation in all patients. In 103 (66\%) patients, antibiotics had been taken within 7 days prior to FOB. The median length of hospital stay was 8 days (range 1-40 days). One patient died within 30 days of admission.

Out of the 51 controls included, 12 were excluded as they were diagnosed by radiography as having pulmonary infection with infiltrates $(n=9)$, lung abscess $(n=1)$, empyema $(n=1)$, and bronchiectasies $(n=1)$. One control was excluded as no BAL culture was performed, and another two controls were excluded as they had taken antibiotics during the preceding 7 days. Of the remaining 36 controls, 22 had lung malignancies and 14 had no pathology identified by FOB or radiological examinations. The median age of the 36 controls was $63 \mathrm{yrs}$ (range 30-77 yrs), and $32(89 \%)$ were current or previous smokers.

\section{Identification of pathogens in patients with LRTI}

In 55 LRTI patients (35\%), any of the four studied pathogens was considered to be the aetiology, i.e. S. pneumoniae in 18 $(12 \%)$ patients, $H$. influenzae in $30(19 \%)$ patients, both $S$. pneumoniae and $H$. influenzae in two $(1.3 \%)$ patients, $M$. pneumoniae in five $(3.2 \%)$ patients, and C. pneumoniae in no patients.

S. pneumoniae was identified by blood culture in six (3.9\%) out of 152 patients tested, by urinary antigen test in nine $(6.3 \%)$ out of 142 , by BAL culture in $10(6.4 \%)$ out of 156 , and by sputum culture in three $(5.3 \%)$ out of 56 . Two of those with positive sputum culture were also BAL-culture positive for $S$. pneumoniae. H. influenzae was identified by BAL culture in $31(20 \%)$ out of 156 patients tested and by sputum culture in six (11\%) out of 56 , but in no blood culture. Five out of the six patients with positive sputum culture were also BAL-culture positive for $H$. influenzae. M. pneumoniae was identified in five patients by both BAL sPCR and throat swab sPCR. BAL sPCR was performed in 154 patients and throat swab sPCR in 156 patients. No patient was positive for C. pneumoniae, although BAL SPCR was performed in 155 patients, throat swab sPCR in 155, BAL culture in 154, and throat swab cultures in 154.

No patient was positive for Legionella spp. by urinary antigen test (154 tested), BAL sPCR (155 tested), or throat swab sPCR (156 tested). No patient was blood- or sputum-culture positive for Staphylococcus aureus or Gram-negative enteric bacilli. However, BAL culture identified S. aureus in 10 patients, Gram-negative enteric bacilli in 10 patients, Moraxella spp. in five patients, Neisseria meningitidis in three patients, and Enterococcus faecalis in one patient. sPCR identified C. psittaci in one patient.

\section{Streptococcus pneumoniae}

S. pneumoniae was an aetiological agent in $22(14 \%)$ patients by the conventional tests, and was identified by BAL mPCR in 44 $(28 \%)$ of the 156 LRTI patients. With all tests included, S. pneumoniae was identified in $30 \%$ of the LRTI patients. 


\begin{tabular}{|c|c|c|c|c|c|}
\hline $\begin{array}{ll}\text { TABLE } 1 & \text { Performance } \\
& \text { tract infectio }\end{array}$ & of multiplex PCR (mPCR) a & d to bronc & lar lavage $(\mathrm{B}$ & 156 adults & er respiratory \\
\hline Species & Reference tests & Sensitivity ${ }^{\#}$ & Specificity & $\mathbf{P P V}^{+}$ & $\mathbf{N P V}^{\S}$ \\
\hline Streptococcus pneumoniae & $\begin{array}{l}\text { Blood culture, BAL culture, sputum } \\
\text { culture and urinary antigen test }\end{array}$ & $86(19 / 22)$ & $81(109 / 134)$ & $43(19 / 44)$ & $97(109 / 112)$ \\
\hline Haemophilus influenzae & $\begin{array}{l}\text { Blood culture, BAL culture and } \\
\text { sputum culture }\end{array}$ & $88(28 / 32)$ & $64(79 / 124)$ & $38(28 / 73)$ & $95(79 / 83)$ \\
\hline Mycoplasma pneumoniae & BAL PCR and throat swab PCR & $100(5 / 5)$ & $100(151 / 151)$ & $100(5 / 5)$ & $100(151 / 151)$ \\
\hline Chlamydophila pneumoniae & $\begin{array}{l}\text { BAL PCR, BAL culture, throat swab } \\
\text { PCR and throat swab culture }\end{array}$ & $0(0 / 0)$ & $99(155 / 156)$ & $0(0 / 1)$ & $100(155 / 155)$ \\
\hline
\end{tabular}

Table 1 demonstrates moderate-to-high sensitivity and negative predictive value, but low specificity and positive predictive value of BAL mPCR for $S$. pneumoniae. Among the six patients with pneumococcal bacteraemia, S. pneumoniae was identified by BAL mPCR in all six patients and by BAL culture in one patient. Likewise, of the nine patients with positive urinary antigen test, $S$. pneumoniae was identified in seven by BAL $\mathrm{mPCR}$ and in none by BAL culture. Among the 87 patients with infiltrates shown by radiography, $\mathrm{MPCR}$ for $S$. pneumoniae had a sensitivity of $94 \%$ (16 out of 17) and a specificity of $73 \%$ (51 out of 70 ).

\section{Haemophilus influenzae}

$H$. influenzae was an aetiological agent in $32(21 \%)$ patients by the conventional tests, and was identified by BAL MPCR in 73 $(47 \%)$ of the 156 LRTI patients. While the sensitivity and negative predictive value of BAL $\mathrm{mPCR}$ for $H$. influenzae were moderate to high, the specificity and positive predictive value were low (table 1). Among the 87 patients with infiltrates shown by radiography, mPCR for $H$. influenzae had a sensitivity of $84 \%$ (16 out of 19) and a specificity of $62 \%$ (42 out of 68 ).

H. influenzae was identified in $20 \%$ by BAL culture and $51 \%$ by BAL mPCR in the 110 current or previous smokers compared with $22 \%$ by BAL culture and $37 \%$ by BAL mPCR (not significant) in the 27 patients who had never smoked.

\section{Mycoplasma pneumoniae}

M. pneumoniae was an aetiological agent in five (3.2\%) LRTI patients. There was total agreement between BAL sPCR, throat swab sPCR and BAL mPCR for identification of M. pneumoniae in the present study (table 1). None of these patients had any other pathogen identified.

\section{Chlamydophila pneumoniae}

No patient had positive sPCR or positive culture for $C$. pneumoniae. The patient with BAL $\mathrm{mPCR}$ positive for $C$. pneumoniae had no throat swab SPCR for $C$. pneumoniae performed, but BAL sPCR and the two C. pneumoniae cultures were negative.

\section{Identification of more than one pathogen}

The relationship between the different tests for S. pneumoniae and $H$. influenzae is shown in table 2. Both of these pathogens were identified in 18 patients as follows: S. pneumoniae, but not H. influenzae, was identified in 29 patients; and H. influenzae, but not S. pneumoniae, was identified in 59 patients. Among the 22 patients with $S$. pneumoniae aetiology, BAL culture was positive for $S$. aureus in two patients and Moraxella spp. in one patient. Two patients with $H$. influenzae aetiology were also BAL-culture positive for Gram-negative enteric bacilli.

\section{Diagnostic influence of antibiotic treatment prior to bronchoscopy}

Among 53 patients with no antibiotics taken prior to FOB, BAL culture and BAL mPCR identified S. pneumoniae in $13 \%(n=7)$ and $23 \%(n=12)$, respectively, and H. influenzae in $19 \%(n=10)$ and $42 \%(n=22)$, respectively, of the patients (nonsignificant). A bacterial concentration of $\geqslant 10^{4} \mathrm{cfu} \cdot \mathrm{mL}^{-1}$ in BAL culture was identified in six out of seven patients identified with S. pneumoniae and in eight out of 10 patients identified with $H$. influenzae.

In the 103 patients with antibiotics taken prior to FOB, BAL culture and BAL mPCR identified S. pneumoniae in $2.9 \%(n=3)$ and $31 \%(n=32)$, respectively $(\mathrm{p}<0.001$, Chi-squared test), and $H$. influenzae in $20 \%(n=21)$ and $50 \%(n=51)$, respectively (nonsignificant), of the patients. A bacterial concentration of $\geqslant=10^{4} \mathrm{cfu} \cdot \mathrm{mL}^{-1}$ in BAL culture was identified in one out of three patients identified with S. pneumoniae and in 16 out of 21 patients identified with $H$. influenzae. One LRTI patient with pneumococcal bacteraemia had taken antibiotics prior to blood sampling and FOB, and S. pneumoniae was identified by BAL mPCR but not by BAL culture in that patient. Of the five patients with $M$. pneumoniae aetiology, three had taken antibiotics prior to FOB.

\section{Bacterial identification in the control group}

Table 3 shows the pathogens identified by BAL culture and BAL mPCR in the control group. Among the 36 controls, either BAL culture or BAL mPCR identified S. pneumoniae in six $(17 \%)$ controls and H. influenzae in 14 (39\%) controls. In the controls with lung malignancy, S. aureus was identified in three controls and Gram-negative enteric bacilli in two controls. S. aureus was also identified in one control without identified pathology. No control was $\mathrm{mPCR}$ positive for either M. pneumoniae or C. pneumoniae.

\section{PCR inhibition}

No PCR inhibition was identified in any BAL mPCR analysis. 
TABLE 2 Combined results of tests for Streptococcus pneumoniae and Haemophilus influenzae in 156 patients with lower respiratory tract infection

\begin{tabular}{|c|c|c|c|c|c|}
\hline \multicolumn{3}{|c|}{ Results regarding S. pneumoniae } & \multicolumn{2}{|c|}{ Results regarding $\boldsymbol{H}$. influenzae } & \multirow{2}{*}{$\begin{array}{l}\text { Patients with the } \\
\text { indicated result } \\
\text { combination noted }\end{array}$} \\
\hline $\begin{array}{l}\text { Blood culture and/or } \\
\text { urinary antigen test }\end{array}$ & $\begin{array}{l}\text { BAL culture and/or } \\
\text { sputum culture }\end{array}$ & BAL $\mathrm{mPCR}^{+}$ & $\begin{array}{l}\text { BAL culture and/or } \\
\text { sputum culture }^{\S}\end{array}$ & BAL mPCR $^{f}$ & \\
\hline
\end{tabular}

\begin{tabular}{|c|c|c|c|c|c|}
\hline+ & - & + & - & - & 6 \\
\hline - & + & - & - & - & 1 \\
\hline+ & + & + & - & + & 1 \\
\hline+ & - & + & + & + & 1 \\
\hline+ & - & + & - & + & 2 \\
\hline - & + & + & - & + & 1 \\
\hline - & - & + & + & + & 3 \\
\hline - & - & + & + & - & 2 \\
\hline - & - & + & - & + & 6 \\
\hline - & - & - & + & + & 23 \\
\hline
\end{tabular}

BAL: bronchoalveolar lavage; mPCR: multiplex PCR; +: positive; -: negative. ${ }^{*}$ : positive, $n=12 ;{ }^{9}:$ positive, $n=11{ }^{+}:$positive, $n=44 ;{ }^{\S}:$ positive, $n=32 ;{ }^{f}$ : positive, $n=73$;

$\# \#$ : the number of patients with the certain combination of results of the first five columns.

\section{DISCUSSION}

In pneumonia, BAL culture has been shown to reliably reflect the microorganisms of the lungs both qualitatively and quantitatively [21, 22], and BAL is the preferred bronchoscopic sample for aetiological diagnosis of LRTI, according to the European Respiratory Society's recently published guidelines for LRTI [3]. In several studies, BAL culture has been used to establish the aetiology of LRTI [5, 23-29].

TABLE 3 Combined results of tests for Streptococcus pneumoniae and Haemophilus influenzae in 36 controls

\begin{tabular}{cc} 
Results regarding S. pneumoniae \\
\hline BAL culture $^{\#} \quad$ BAL mPCR
\end{tabular}

\begin{tabular}{|c|c|c|c|c|c|}
\hline & BAL culture ${ }^{\#}$ & BAL $\mathrm{mPCR}$ & BAL culture $^{+}$ & BAL $\mathrm{mPCR}^{\S}$ & resuit combination noted \\
\hline \multirow{5}{*}{$\begin{array}{l}\text { Controls with lung } \\
\text { malignancy } \# \#\end{array}$} & - & + & - & - & 1 \\
\hline & + & + & - & + & 1 \\
\hline & + & - & + & + & 1 \\
\hline & - & - & + & + & 3 \\
\hline & - & - & - & + & 5 \\
\hline \multirow{4}{*}{$\begin{array}{l}\text { Controls with no } \\
\text { pathology } \\
\text { identified" }\end{array}$} & - & + & - & - & 1 \\
\hline & + & - & - & + & 1 \\
\hline & - & + & - & + & 1 \\
\hline & - & & - & + & 2 \\
\hline
\end{tabular}

BAL: bronchoalveolar lavage; mPCR: multiplex PCR; +: positive; -: negative. ${ }^{\#}$ : positive, $n=3 ;{ }^{\circ}$ : positive, $n=4 ;^{+}$: positive, $n=4{ }^{\text {s }}$ : positive, $n=14$; ${ }^{f}$ : number of patients with the certain combination of results of the previous four columns; $\#: n=22 ;{ }^{*}: n=14$. 
BAL samples collected by FOB can potentially be contaminated with the oropharyngeal flora by the bronchoscope itself and can cause false positive microbiological results [30, 31]. However, in populations with low rates of carriage in the oropharynx of pathogenic bacteria, some contamination from the oropharyngeal flora may be unimportant. In two previous studies of BAL culture in healthy adults with 15 and 14 subjects, respectively [27, 32], no S. pneumoniae or H. influenzae could be identified at the detection level of $10^{4} \mathrm{cfu} \cdot \mathrm{mL}^{-1}$. KIRKPATRICK et al. [31] found $H$. influenzae at a concentration of $10^{1} \mathrm{cfu} \cdot \mathrm{mL}^{-1}$ in one out of eight healthy subjects, and $S$. pneumoniae in none. This indicates the high specificity of BAL culture for identification of S. pneumoniae and H. influenzae in LRTI in populations with a low carriage rate of these bacteria. In lower respiratory tract specimens, S. pneumoniae and $H$. influenzae are more sensitive to antibiotics than many other bacteria [4]. Thus, in patients treated with antibiotics, it seems reasonable to use a detection level for these two bacteria of $<10^{4} \mathrm{cfu} \cdot \mathrm{mL}^{-1}$. Among the 53 patients not pre-treated with antibiotics in the present study, S. pneumoniae or H. influenzae was identified at a concentration of $\geqslant 10^{4} \mathrm{cfu} \cdot \mathrm{mL}^{-1}$ in $14(26 \%)$ cases and $10^{2}-10^{3} \mathrm{cfu} \cdot \mathrm{mL}^{-1}$ in three $(5.7 \%)$ cases. Due to the low frequency in the latter, a detection level of $10^{2} \mathrm{cfu} \cdot \mathrm{mL}^{-1}$ was used for BAL culture as well as BAL mPCR in all cases in the study.

In LRTI patients with ongoing antibiotic treatment, BAL mPCR was significantly more often positive for S. pneumoniae than BAL culture. The present authors have previously demonstrated that the present urinary antigen test is more often positive in those pre-treated than in those not pre-treated with antibiotics [14]. Thus, mPCR and the urinary antigen test appear useful in patients treated with antibiotics, in order to identify S. pneumoniae in patients who will probably give false negative results in their cultures. As FOB is predominantly performed because of treatment failure, BAL $\mathrm{MPCR}$ can be a useful routine complement to BAL culture. In the present study, FOB was performed on an unselected LRTI population. However, $66 \%$ of the patients had taken antibiotics prior to FOB, and many of them had experienced failure to respond to the outpatient antibiotic treatment, although treatment failure was not defined by the study protocol.

The high rate of antibiotic treatment prior to collection of samples and the low frequency of $S$. pneumoniae aetiology established $(14 \%)$ in the present study are probably the major reasons for the suboptimal specificity of $\mathrm{MPCR}$ for S. pneumoniae. Difficulty to estimate reliable specificity is a general problem for new diagnostic tests that are more sensitive than the reference methods. If an SPCR for S. pneumoniae had been included in the reference standard, the specificity of mPCR for S. pneumoniae would probably have been higher and more correct. However, the usefulness of mPCR for S. pneumoniae was supported by the low number of positives in the control group.

When predictive values are calculated for a test, the incidence of the investigated disease is crucial for the level of these values. In a review article of lung aspiration in CAP, SCOTT and HALL [1] found that $48 \%$ of adult CAP patients were positive for S. pneumoniae in blood culture and/or lung aspirate culture. If the $\mathrm{mPCR}$, with its current sensitivity and specificity for
LRTI, was used in a LRTI population with a true frequency of S. pneumoniae aetiology of $48 \%$, the positive predictive value would be $81 \%$ and the negative predictive value would be $87 \%$.

Although the sensitivity was rather high, the specificity of BAL mPCR for $H$. influenzae was low in the present study. PCR has previously been shown to identify a high rate of colonisation of $H$. influenzae in patients with chronic obstructive pulmonary disease [33], but $H$. influenzae was also identified in $22 \%$ by BAL culture and $37 \%$ by BAL mPCR in 27 patients who had never smoked. As mPCR has shown a high analytical specificity for $H$. influenzae [12], and as sputum MPCR was positive for $H$. influenzae in only two $(7.7 \%)$ out of 26 CAP patients with definite aetiologies other than $H$. influenzae in the present authors' previous study [13], the high rate of $H$. influenzae in the present study probably represents colonisation. Hence, it would be interesting to test mPCR on BAL samples from another LRTI population and another control population with a lower smoking frequency.

Owing to a low frequency of $C$. pneumoniae infection during the study period, the sensitivity of BAL $\mathrm{MPCR}$ for $C$. pneumoniae could not be evaluated. In the present authors' previous study of 235 patients with CAP [13], three patients were microimmunofluorescence-test positive for C. pneumoniae in paired sera; these three patients were also mPCR positive for $C$. pneumoniae in nasopharyngeal secretions. The fact that no control and only one LRTI patient was BAL mPCR positive in the present study indicates that BAL $\mathrm{MPCR}$ is probably specific for $C$. pneumoniae. BAL $\mathrm{mPCR}$ should be tested on another LRTI population with a higher incidence of $C$. pneumoniae infection to evaluate the usefulness of this assay. BAL mPCR demonstrated an optimal performance for M. pneumoniae in the present study (table 1).

Atypical respiratory pathogens have been identified by several previous MPCR protocols [34-37]. However, the present PCR protocol is unique, as it is constructed for identification of two typical and two atypical pathogens, probably the four most common aetiologies of CAP [38].

Due to the high documented aetiological frequencies of the four studied pathogens and due to the high negative predictive values of BAL MPCR (table 1), negative MPCR results may be used to rule out these pathogens as aetiological agents. For a BAL sample that is MPCR positive for a single pathogen, the negative results of the three other pathogens increase the likelihood that the positive test result is truly positive.

As rather low frequencies of $S$. pneumoniae and no $M$. pneumoniae or $C$. pneumoniae were identified in the control group, BAL mPCR results positive for any of these three pathogens can support their role as aetiological agents in LRTI. Consequently, mPCR can be used to support therapeutic decisions in patients with LRTI.

In conclusion, bronchoalveolar lavage multiplex PCR appears to be a useful aetiological tool in lower respiratory tract infection patients, particularly in patients treated with antibiotics. This method could be a valuable supplement to bronchoalveolar lavage culture. 


\section{ACKNOWLEDGEMENTS}

The authors would like to thank B. Ekström for skilful technical assistance.

\section{REFERENCES}

1 Scott JA, Hall AJ. The value and complications of percutaneous transthoracic lung aspiration for the etiologic diagnosis of community-acquired pneumonia. Chest 1999; 116: $1716-1732$.

2 Hedlund J, Strålin K, Örtqvist A, , Holmberg H, the Community-Acquired Pneumonia Working Group of the Swedish Society of Infectious Diseases, Swedish guidelines for the management of community-acquired pneumonia in immunocompetent adults. Scand J Infect Dis 2005; 37: 791-805.

3 Woodhead M, Blasi F, Ewig S, et al. Guidelines for the management of adult lower respiratory tract infections. Eur Respir J 2005; 26: 1138-1180.

4 Prats E, Dorca J, Pujol M, et al. Effects of antibiotics on protected specimen brush sampling in ventilator-associated pneumonia. Eur Respir J 2002; 19: 944-951.

5 Hohenthal U, Sipila J, Vainionpaa R, et al. Diagnostic value of bronchoalveolar lavage in community-acquired pneumonia in a routine setting: a study on patients treated in a Finnish university hospital. Scand J Infect Dis 2004; 36: 198-203.

6 Chastre J, Combes A, Luyt CE. The invasive (quantitative) diagnosis of ventilator-associated pneumonia. Respir Care 2005; 50: 797-812.

7 Wheeler J, Freeman R, Steward M, et al. Detection of pneumolysin in sputum. J Med Microbiol 1999; 48: 863-866.

8 Murdoch DR. Molecular genetic methods in the diagnosis of lower respiratory tract infections. APMIS 2004; 112: 713-727.

9 Dorigo-Zetsma JW, Verkooyen RP, van Helden HP, van der Nat H, van den Bosch JM. Molecular detection of Mycoplasma pneumoniae in adults with communityacquired pneumonia requiring hospitalization. J Clin Microbiol 2001; 39: 1184-1186.

10 Raty R, Ronkko E, Kleemola M. Sample type is crucial to the diagnosis of Mycoplasma pneumoniae pneumonia by PCR. J Med Microbiol 2005; 54: 287-291.

11 Kuoppa Y, Boman J, Scott L, Kumlin U, Eriksson I, Allard A. Quantitative detection of respiratory Chlamydia pneumoniae infection by real-time PCR. J Clin Microbiol 2002; 40: 2273-2274.

12 Strålin K, Bäckman A, Holmberg H, Fredlund H, Olcén P. Design of a multiplex PCR for Streptococcus pneumoniae, Haemophilus influenzae, Mycoplasma pneumoniae and Chlamydophila pneumoniae to be used on sputum samples. APMIS 2005; 113: 99-111.

13 Strålin K, Törnqvist E, Kaltoft MS, Olcén P, Holmberg H. Etiologic diagnosis of adult bacterial pneumonia by culture and PCR applied to respiratory tract samples. J Clin Microbiol 2006; 44: 643-645.

14 Korsgaard J, Møller JK, Kilian M. Antibiotic treatment and the diagnosis of Streptococcus pneumoniae in lower respiratory tract infections in adults. Int J Infect Dis 2005; 9: 274-279.
15 Rasmussen TR, Korsgaard J, Møller JK, Sommer T, Kilian M. Quantitative culture of bronchoalveolar lavage fluid in community-acquired lower respiratory tract infections. Respir Med 2001; 95: 885-890.

16 Murray PR, Baron EJ, Pfaller MA, Tenover FC, Yolken RH, eds. Manual of Clinical Microbiology. 7th Edn. Washington, D.C., ASM Press, 1999.

17 Storgaard M, Østergaard L, Jensen JS, et al. Chlamydia pneumoniae in children with otitis media. Clin Infect Dis 1997; 25: 1090-1093.

18 Tarp B, Jensen JS, Østergaard L, Andersen PL. Search for agents causing atypical pneumonia in HIV-positive patients by inhibitor-controlled PCR assays. Eur Respir J 1999; 13: 175-179.

19 Farholt S. Chlamydia pneumoniae. PhD thesis. University of Copenhagen, Copenhagen, Denmark, 1996.

20 Nielsen SV, Henrichsen J. Detection of pneumococcal polysaccharide antigens in the urine of patients with bacteraemic and non-bacteraemic pneumococcal pneumonia. Zentralbl Bakteriol 1994; 281: 451-456.

21 Chastre J, Fagon JY, Bornet-Lecso M, et al. Evaluation of bronchoscopic techniques for the diagnosis of nosocomial pneumonia. Am J Respir Crit Care Med 1995; 152: 231-240.

22 Johanson WG Jr, Seidenfeld JJ, Gomez P, de los Santos R, Coalson JJ. Bacteriologic diagnosis of nosocomial pneumonia following prolonged mechanical ventilation. Am Rev Respir Dis 1988; 137: 259-264.

23 Sörensen J, Forsberg P, Håkanson E, et al. A new diagnostic approach to the patient with severe pneumonia. Scand J Infect Dis 1989; 21: 33-41.

24 Thorpe JE, Baughman RP, Frame PT, Wesseler TA, Staneck JL. Bronchoalveolar lavage for diagnosing acute bacterial pneumonia. J Infect Dis 1987; 155: 855-861.

25 Feinsilver SH, Fein AM, Niederman MS, Schultz DE, Faegenburg DH. Utility of fiberoptic bronchoscopy in nonresolving pneumonia. Chest 1990; 98: 1322-1326.

26 Jimenez P, Saldias F, Meneses M, Silva ME, Wilson MG, Otth L. Diagnostic fiberoptic bronchoscopy in patients with community-acquired pneumonia. Comparison between bronchoalveolar lavage and telescoping plugged catheter cultures. Chest 1993; 103: 1023-1027.

27 Dalhoff K, Braun J, Hollandt H, Lipp R, Wiessmann KJ, Marre R. Diagnostic value of bronchoalveolar lavage in patients with opportunistic and nonopportunistic bacterial pneumonia. Infection 1993; 21: 291-296.

28 Korsgaard J, Rasmussen TR, Sommer T, Møller JK, Jensen JS, Kilian M. Intensified microbiological investigations in adult patients admitted to hospital with lower respiratory tract infections. Respir Med 2002; 96: 344-351.

29 van der Eerden MM, Vlaspolder F, de Graaff CS, Groot T, Jansen HM, Boersma WG. Value of intensive diagnostic microbiological investigation in low- and high-risk patients with community-acquired pneumonia. Eur J Clin Microbiol Infect Dis 2005; 24: 241-249.

30 Halperin SA, Suratt PM, Gwaltney JM Jr, Groschel DH, Hendley JO, Eggleston PA. Bacterial cultures of the lower respiratory tract in normal volunteers with and without experimental rhinovirus infection using a plugged double catheter system. Am Rev Respir Dis 1982; 125: 678-680.

31 Kirkpatrick MB, Bass JB Jr. Quantitative bacterial cultures of bronchoalveolar lavage fluids and protected brush 
catheter specimens from normal subjects. Am Rev Respir Dis 1989; 139: 546-548.

32 Cabello H, Torres A, Celis R, et al. Bacterial colonization of distal airways in healthy subjects and chronic lung disease: a bronchoscopic study. Eur Respir J 1997; 10: 1137-1144.

33 Murphy TF, Brauer AL, Schiffmacher AT, Sethi S. Persistent colonization by Haemophilus influenzae in chronic obstructive pulmonary disease. Am J Respir Crit Care Med 2004; 170: 266-272.

34 Welti M, Jaton K, Altwegg M, Sahli R, Wenger A, Bille J. Development of a multiplex real-time quantitative PCR assay to detect Chlamydia pneumoniae, Legionella pneumophila and Mycoplasma pneumoniae in respiratory tract secretions. Diagn Microbiol Infect Dis 2003; 45: 85-95.
35 Pinar A, Bozdemir N, Kocagoz T, Alacam R. Rapid detection of bacterial atypical pneumonia agents by multiplex PCR. Cent Eur J Public Health 2004; 12: 3-5.

36 Tong CY, Donnelly C, Harvey G, Sillis M. Multiplex polymerase chain reaction for the simultaneous detection of Mycoplasma pneumoniae, Chlamydia pneumoniae, and Chlamydia psittaci in respiratory samples. J Clin Pathol 1999; 52: 257-263.

37 Corsaro D, Valassina M, Venditti D, Venard V, Le Faou A, Valensin PE. Multiplex PCR for rapid and differential diagnosis of Mycoplasma pneumoniae and Chlamydia pneumoniae in respiratory infections. Diagn Microbiol Infect Dis 1999; 35: 105-108.

38 Marrie TJ. Etiology of community-acquired pneumonia. In: Marrie TJ, ed. Community-Acquired Pneumonia. New York, Kluwer Academic/Plenum Publishers, 2001; pp. 131-141. 